\title{
CheckOff-M: Model Checking and Its Role in IP
}

\author{
Roger B. Hughes \\ Abstract, Inc. \\ 47211 Lakeview Boulevard, \\ Fremont, CA 94538-6530 USA
}

\begin{abstract}
CheckOff-M is a formal verification tool which performs model checking. Model checking allows you to find errors in a design and to verify critical properties, such as the absence of deadlocks and whether the design performs specified functions. You provide a set of logical properties which the design should possess, and CheckOff-M tells you if the design omits required behavior or includes unwanted behavior. For example, you can check that (i) mutually exclusive events cannot occur concurrently, or (ii) a desired event will occur at a specific time.

CheckOff-M can model check both complex combinatorial and sequential designs, and you can use CheckOff-M at all stages of your design flow because CheckOff-M can check behavioral, register-transfer level and gate-level designs. CheckOff-M works with VHDL, EDIF and Verilog designs, so you can easily integrate CheckOff-M with your existing design flow.

CheckOff-M represents designs internally using efficient implementations of Ordered Binary Decision Diagrams (OBDDs).
\end{abstract}

CIFIP 1997. Published by Chapman \& Hall 